\title{
Dissociative Disorders with Haemolacria: Series of Case Reports
}

\author{
MS RAHMAN $^{\mathrm{a}}$, MR KARIM $^{\mathrm{b}}$, MM ISLAM $^{\mathrm{c}}$, MR KARIM $^{\mathrm{d}}$
}

\begin{abstract}
Summary:
Bloody tears (Haemolacria) are a rare symptom that can be caused by local or systemic pathology. It is one of the most alarming symptom in ophthalmology. Besides those, idiopathic cases have been reported. A case of hyperthyroidism where haemolacria was secondary to the condition has also been reported. Haemolacria are also reported as secondary to epistaxis. Psychogenic causes are described including Munchausen Syndrome by proxy. Here we describe a series of four cases of haemolacria along with bleeding from other sites, found as associated features of dissociative disorders. In this series, patients with age ranging from 14-17 years, three of them are female and one male have been included.
\end{abstract}

Introduction

Hemolacria is a rare clinical condition, literally meaning "bloody tears". In the literature, it has been reported in cases with conjunctival capillary hemangioma, conjunctival telangiectasias, bacterial conjunctivitis, lacrimal sac tumors, lacrimal sac infections, nasal and paranasal sinus tumors ${ }^{1}$. There are cases of idiopathic haemolacria with no identified causes have been reported ${ }^{2}$. A case of bloody tears in a hyperthyroid female patient who displayed no evidence of localized ocular pathology has been reported ${ }^{4}$. Haemolacria has been described in Hereditary hemorrhagic telangiectasia ${ }^{5}$, Henoch-Schönlein purpura and

a. Dr. Md. Shafiqur Rahman, Associate Professor, Department of Psychiatry, Sylhet Women's Medical College

b. Professor Md. Rezaul Karim, Professor and Head, Department of Psychiatry and Principal, Sylhet Women's Medical College

c. Dr. Mohammed Misbahul Islam, Registrar, Department of Psychiatry, Sylhet Women's Medical College

d. Dr. Md. Redwanul Karim, Consultant Psychiatrist, Department of Psychiatry, Sylhet Women's Medical College Hospital

Address of correspondence: Dr. Md. Shafiqur Rahman, Associate Professor of Psychiatry, Sylhet Women's Medical College, Mirboxtula, Sylhet-3100, Bangladesh. E-mail: shafdoc@yahoo.com

Received: 6 Oct. 2016

Accepted: 3 Nov. 2016
Examination excluded local ocular and nasal pathology, coagulopathy and hyperthyroidism. In course of their illness, two of these cases met the DSM 5 criteria for both dissociative disorders and conversion disorders, rest of them have been diagnosed as mixed dissociative disorders. After appropriate intervention, three patients recovered completely and in one patient symptoms (also bleeding) recurred on re-exposure to the previous stress factors.

We report three cases of Dissociative disorders and one with both dissociative and conversion disorder where bloody tears were one of the feature. To the best of our knowledge this is the first official report of its kind in Bangladesh.

(J Bangladesh Coll Phys Surg 2017; 35: 36-42)

retrograde epistaxis and in women during menstrual period. Cases of 'bloody tears' in a hysterical young female ${ }^{1}$, during episodes of cluster headache in a case of Gardner-Diamond syndrome ${ }^{6}$ have been reported as well. Psychogenic purpura consists in the spontaneous appearance of recurrent bruising, is an unexplained reality which, in addition to cutaneous ecchymoses, may have bleeding from multiple sites, like, haemolacria along with haematuria, menometrorrhagia, GIT bleeding and epistaxis. There may be spontaneous psychogenic as well as self-inflicted iatrogenic bleeding. There is virtually no scientific information on the interaction between the nervous system and hemostasis or blood coagulation $^{5}$. It is remarkable that blood coagulation and other hemostatic tests remain normal in all patients of psychogenic bleeding, including haemolacria. Other bleeding disorders with normal coagulation tests are an inherited failure of collagen biosynthesis or congenital or acquired vascular disorders, but no note of haemolacria as a sign in those disorders has been found ${ }^{12}$. Although in some literatures, haemolacria has been noted as a symptom of 'Hysteria' and other 'Psychological' illnesses ${ }^{1,2,5}$, This sign has not been categorically included in any particular psychiatric disorder.

\section{Case 1}

A 16 years old male student of class X, from Sylhet City, admitted in Sylhet Women's Medical college Hospital 
in the department of psychiatry on $1 / 6 / 2012$ with the complaints of episodes of bloody tears from both eyes (haemolacria), bleeding from mouth, bleeding per urethra, chest pain and severe convulsion and inability to talk.

History revealed that the patient was alright about two years ago. Since then he had had multiple episodes of abnormalities characterized by unresponsiveness, convulsions, loss of power in the limbs, inability to hear and see and bleeding from the nose and eyes. According to the parents, first he developed difficulty in breathing and stiffness in both limbs followed by convulsion and paralysis of the lower limbs. Likely precipitating factor was the death of a cousin to whom he was very much attached. That was a single episode relieved after sometime with medical intervention. On another occasion, patient developed pricking sensation on the left side of his body followed by breathlessness, on receiving treatment from a nearby hospital he became symptom free after 24 hours. Since then episodes with different types of features have been continued off and on. About one and half months from the last episode, he started to bleed through mouth. Sometimes he cut his hands to bleed. His schooling hampered seriously and became irregular.

The patient had been evaluated by multiple doctors of different specialties. No physical abnormalities were detected. He was also seen by psychiatrist and clinical psychologist before admission in the hospital.

His father is a businessman, mother house wife; lives in an extended family with his parents, a brother and two sisters along with uncle, aunt and cousins. High expressed emotions within the family members noted from history. No consanguinity within the parents. The family is economically solvent. There is no history of psychiatric disorders among $1^{\text {st }}$ degree relatives.

Patient's birth and early development was normal but in childhood, he used to show temper tantrums and increasingly became demanding, easily gets hurt. Patient complains of apparent lack of appreciation from his parents.

Relation with his siblings is mostly friendly, parents are over caring and classmates are cooperative. He lacks a sense of humor and unable to cope with the sarcastic remarks of his classmates and sometimes become irritated. He is polite, gets easily hurt, and very much sensitive; most of the time he remains depressed, and always tries to find a negative meaning of any remark made by his parents and peers. His performance was mediocre in the school. He has no hobby, passes the leisure time by walking around the house or sitting idle.

Past medical and psychiatric history is nothing contributory. No history suggestive of seizure disorder, mood disorder, anxiety symptoms or PTSD or Malingering. Factitious Disorder also excluded as per DSM 5 criteria.

General and systemic physical examination revealed normal findings. His mental state was otherwise normal except that he was found distracted most of the time during interview.

He complained of poor memory and thinks that he has no disease excepting sometimes his lower limbs become stiff and he lives with sorrow and pain.

All of his lab investigations on different occasions at different hospitals revealed normal findings which include: CBC, Calcium level, USG whole abdomen, CXR, Endoscopy of upper GIT, BT, CT, CT scan of the brain, MRI of the brain, S. bilirubin, AST, ALT, S. alkaline phosphatase, S. protein, urea, S. creatinine, phosphate, LDH, TFT, Urinalysis, APTT, S.C3, C4, ANA, CRP, Anti DS DNA, RF.

Patient is diagnosed as Mixed Dissociative Disorder.

Low dose antidepressants and anxiolytics have been used during his stay in the hospital and counselling continued. Improvement was minor and patient's parents wanted discharge for social reason.

As desired by his family, patient was referred to the National Institute of Mental Health and Neurosciences (NIMHANS), Bangalore, India, for further evaluation and management, where he was treated as inpatient for three months. During his stay, the patient was started on individual therapy addressed intra-psychic conflicts, behavioral principles used to improve concentration and family therapy. Low dose antidepressant and anxiolytic used for a short period. Two other follow up visits every 6 months have been made.

Patient have been found stable without recurrences of features on a single follow up visit after 1 year in our hospital.

\section{Case 2 (Fig.-1)}

A 17 years old unmarried girl from a township of Sylhet, admitted in the department of psychiatry of Sylhet 


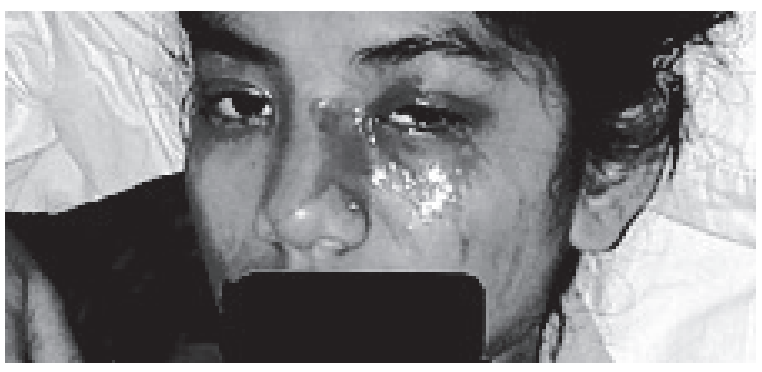

Fig.-1

Women's Medical College on 30/8/2012 with the complaints of episodes of flowing of tear (haemolacria) from both eyes, abnormal abdominal movement followed by blood vomiting after food and paroxysms of abdominal pain for about $2 \frac{1}{2}$ years; episodes of convulsions and loss of consciousness and could not remember anything after recovery. These features, episodic in nature, lasted for about $4 \frac{1}{2}$ years.

Patient stated that she was reasonably well about four years ago. At the outset, she started episodes of dizziness, headache and vomiting which lasted for about one and half months. After few days when she was a student of class VII (2007), one evening she had seen a small boy climbing in a bamboo in a nearby bush, on other occasion she saw a stranger milking a cow in their premises. As stated by the informant (maternal aunt), since then she used to have transient abnormal experiences, like hearing song or sudden single voice while nobody was around her, or as if somebody was coming to kill her. After About 2 months of marriage of her elder sister in mid-2008, changes in her behavior was noted by relatives; like loss of appetite, vomiting after meal, sometimes became increasingly demanding and aggressive. Whenever she wanted something, if not met with, she started screaming, talked non understandable languages, fell on the ground followed by loss of consciousness for a brief period.

She was very much shy since childhood but after development of above features, she became more extroverted and demonstrative, used to spend more time on grooming, (e.g. changing hair style frequently, putting on colors, plucking brow and manicure). She spent most of her time by watching TV or with her cell phone. She tries to act like TV Models, videos herself along with other family members. Her father works abroad, mother is a house wife, no consanguinity present. She has two sisters and no brother. She lived in a well to-do extended family along with her parents, grandparents, uncle, aunt and cousins and has very strong attachment with her mother. Born with low birth weight (?), achieved usual milestones, shy and reserved during childhood. Schooling was difficult as she has to walk alone about a kilometer to reach.

So when she was at class $\mathrm{V}$, she left home to her maternal grandparents' house where school was nearer. Although her elder sister had been there since before, she missed her mother too much. When she was a student of class VIII, she started to see glimpses of things which nobody else can see.

She suffered from measles in her childhood. Infrequent migraine attacks on exposure to direct sunlight. No history of any other major medical illness is obtained.

Within her family, most of the time she remained happy and cheerful, passes leisure time watching TV. Her relationship was intense with her mother, aunt and maternal uncle. General examination revealed normal findings.

Among other examination, CBC, BT, CT, Endoscopy upper GIT, S. Electrolytes, S. Creatinine, ECG, CXR, and TSH, were within normal limits.

Patient was diagnosed as having mixed Dissociative Disorder and Migraine attacks. Factitious disorder, Malingering, Psychotic disorders and other differentials excluded.

Anxiolytics and low dose antidepressants used for short period. Interpersonal psychotherapy with explanation of her problems discussed targeting the patient and family.

Patient maintained irregular follow up at OPD up to 2 years without further sequel.

\section{Case 3 (Fig.-2)}

A 14 years old girl of $8^{\text {th }}$ grade, presented at psychiatric OPD of Sylhet Women's Medical College Hospital 19/ $05 / 2014$, with the complaints of episodes of burning sensation all over the body followed by bizarre behavior and unconsciousness, episodic bleeding through mouth, eyes and ears; self-mutilating behaviorparticularly chewing of lower lip, and stiffness in the limbs; for about 2 months. All the symptoms started after a familial stress. 


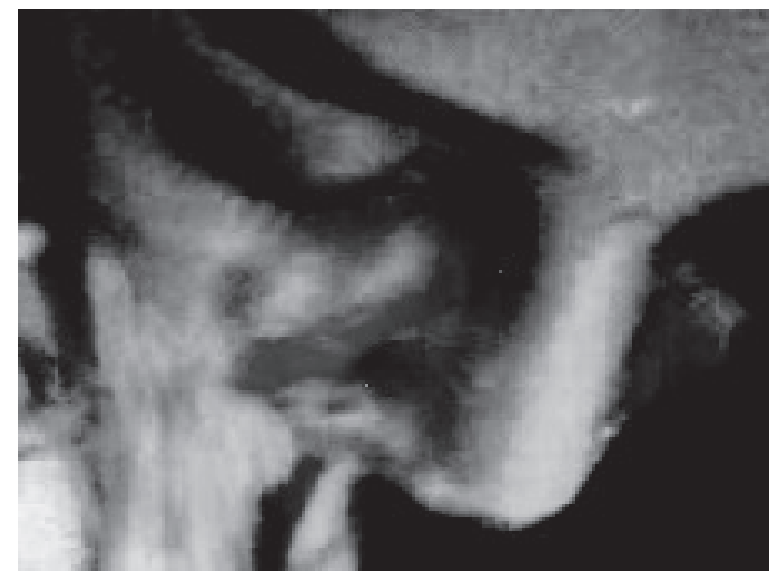

Fig.-2

As per statement of the patient and informants, she was reasonably well 7-8 years ago. When she was 6 years old, she suffered episodic respiratory distress, which was continued despite treatment. For about last 2 years, she has been suffering these episodes with increasing frequency. Moreover, for each episode new features were developed including bizarre behavior, like, tearing apart her clothes, breaking of household things, extreme agitation, running away from the house, biting herself and people around her, followed by brief unconsciousness when sometimes blood coming up through her mouth and both eyes. Each episode lasted for about 40 minutes and after regaining consciousness, patient could not remember anything regarding her behavioral abnormalities. With time she started bleed through her ear and eyes periodically without any other symptom. It was noted that her elder sister, used to have fits even with mild stress, when the patient also got the same by seeing her sister unconscious.

Her father is a businessman, step mother is a house wife. She has 2 sisters and 1 brother. No history of consanguinity between parents, neither any psychiatric illness among her $1^{\text {st }}$ or $2^{\text {nd }}$ degree relatives was obtained. Her birth was domiciliary. Mother died of cancer when she was 9 months old. Since then she has been living with her maternal aunt and brought up in an extended family along with her Grandparents, 2 sisters, uncle and aunt and cousins.

Early development was normal with no abnormal traits. Schooling was uneventful. Age of menarche was at 11. Family environment is otherwise favorable except overindulgence of superstitions. Social circumstances is also good. She had pneumonia in her childhood but no documents were obtained. Since her childhood she maintained a good relationship with her family members and other associates, always cheerful and fond of grooming beautifully. Leisure activity seems to be limited to watching TV. Currently she was asked by her father to shift from her grandparent's family to join her own family but she is unwilling to leave her aunt and live with her step mother.

General physical examination was revealed normal findings. Mental state exam was also normal, anxiety features are noted.

Routine laboratory examinations with BT, CT, were within normal limits.

\section{Diagnosis- Mixed Dissociative Disorder}

Patient has been treated with low dose antidepressants. Nature of her illness explained to the patient and family. She became symptom free within one month and remained so for about a year.

On re exposure to the foregoing family stressor, her symptoms recurred. She has been admitted on 31/05/ 2015 in the psychiatry department. Feigning or factitious disorder excluded by clinical assessment.

Patient received anxiolytics and inter personal psychotherapy therapy along with family therapy and discharged with improvement.

\section{Case-4}

A young 15 years old female student of class- IX from a semi urban area of Sylhet district was admitted 01/06/ 2014 in the psychiatry department of Sylhet Women's Medical College Hospital with the presenting complaints of episodic nose bleeding, bleeding through both ears and decreased sleep. All for about 8 months; episodes of fits for about 10 months; headache for 2 years.

History reveals that patient was in good health 2 year ago. When she was a student of class-VII, once she had an accident while travelling in tricycle (Rickshaw). Since then she had headaches off and on till now. She noticed unexplained change in her mind since then. When she was a student of class-VIII, her brother passed SSC examination and migrated to Sylhet city for higher study, she started to feel lonely as her brother was her 
only playmate and friend within the house. Although her parents were willing to migrate to Sylhet city with all other members, she refused to leave her house mainly because her old friends. Gradually she became more demanding, easily gets angry and impulsive. The patient blamed her mother of not to be sympathetic enough to understand her. When she becomes sick her mother use to tell her of negative social impact of her illness which hurt her so much that sometimes she considered suicide. Due to strong religious conviction and considering her family status in the society, she refrained herself from committing so. Regarding fits she stated that whenever she gets hurt she used to cry which gets her a headache, when she read for a long time she gets headache, exposure to excessive heat also brings headache. Whenever she gets a headache irrespective of cause, she at first feels dizzy, then dimness and blurring of vision and loss of hearing, until she loses consciousness.

As her parent state, then she bleeds through nose and ears and recovers after a brief period. The patient's father is an established businessman, mother is a housewife. She has 3 sisters and 1 brother. No history of consanguinity between parents. Her family holds a middle class status. No history of mental illness in the family, but a cousin of her has been suffering from Bipolar Disorder. Her birth was domiciliary and early development was normal. Since childhood she was demanding and showed heightened reactivity to stress. Schooling and education was uneventful. Her age of menarche was at 11 years. She was born in an extended family but brought up in nuclear one.

Past medical and psychiatric history is normal. She used to maintain good and stable relationship with peer group as well as with her family members, especially with her brother and elder sister but somewhat reserved with her mother. She was always cheerful, with an up to the mark living standard. Her free time activities were limited to mobile gaming and occasionally reading books.

General physical examinations and neurological examinations are normal. ENT examination done previously was also normal. Routine laboratory tests including BT, CT, revealed normal findings.

Patient was diagnosed as Conversion Disorder and Migraine with anxiety features. Any other medical and ENT problems excluded after consulting respective specialties.
After discharge from the hospital the patient did not come back for scheduled follow up but her father responded over telephone. Patient remained symptom free for 1 year.

\section{Discussion:}

Bloody tears or haemolacria is an inexplicable and alarming condition to the physician, patient and guardian. Haemolacria literally means bloody tears, either frank blood and/or blood mixed discharge from one or both eyes. In the literature, this condition have been reported in the patients of different disciplines of medicine. Historically haemolacria could be traced back to the medieval 'stigmatists' of catholic faith, where bleeding from different wound sites (locations corresponding to the crucifixion wounds of Jesus Christ). Stigmata could take various forms including bloody tears ${ }^{9,10}$. In ophthalmology, sanguineous discharge is a relatively frequent finding in acute conjunctival hyperaemia and inflammation but occurrence of bleeding from eyes in isolated form is unusual and extremely rare. Many causes are outlined by different authors; like, conjunctivitis, capillary haemangioma, conjunctival telangiectasia, lacrimal sac tumours or traumma. Ahwalia et al reported case of haemolacria in a young female where episodes of blood tearing was only under stress, like house examinations. Presence of hysterical trait was confirmed by the consulting psychiatrist. We found mutiple stressors working in our cases at the outset of symptoms. M F Wiese reported a 56 years old woman presented with the history of bleeding from the right nostril. When she had tried to stop it by pinching her nose, she then experienced bleeding from her right eye and ear. Bleeding was ultimately found to be due to haemorrhagic telangiectasia inside nasal cavity ${ }^{7}$. Cases of haemolacria secondary to other ENT causes has also been found in literature which include nasal and paranasal tumours. Ozcan KM reported an 11 years old paediatric patient with the complaints of bilateral haemolacria along with recurrent epistaxis where examination revealed a right side deviated nasal septum and increased hyperemic vascularity in the nasal mucosa, other ENT examinations were normal ${ }^{3}$. A case of haemolacria and epistaxis secondary to hyperthyroidism has been reported by Jason et al where local pathology and coagulopathy have been excluded by examination. Symptoms resolved after appropriate treatment ${ }^{4}$. Ho et al reported a series 
of four cases, one boy and three girls ranging 6-14 years where workup finding were normal. In all cases haemolacria resolved without any sequelae over a follow up period of 9-11 months; those cases were reported as idiopathic ${ }^{2}$. Oyenusi and Ananti reported two cases of bloody tears, first one, a boy, presented with cough and bloody tears, the bleeding stopped after routine treatment. The second case was a girl, presented with redness and yellowish mucoid discharge from both eyes with low grade fever for three days followed by bloody tears; this patient was finally diagnosed as orbital cellulitis and managed with antibiotics ${ }^{8}$. M Versetrate wrote in his paper regarding psychogenic bleeding. Psychogenic purpura (Auto-erythrocyte sensitisation) consists in the spontaneous appearance of recurrent bruising, which is still unexplained. Most often woman with an underlying emotional disorder are affected. In addition to cutaneous ecchymoses and hematomas, there may be many other complaints encompassing multiple organ systems along with menorrhagia, metrorrhagia, haematuria, epistaxis and gastrointestinal bleeding and haemolacria. Spontaneous psychogenic cutaneous bruising is very difficult to distinguish from self-inflicted iatrogenic bleeding. The only therapeutic approach in patients with psychogenic bleeding is psychiatric, with particular attention to the sociocultural background of the patient and his family 5,12 . In our report, four cases of haemolacria along with bleeding from multiple sites associated with psychiatric disorders are described. After meticulous history, direct observation, mental state examinations, examinations to exclude local pathology in collaboration with other departments and work-up for all relevant laboratory investigations, three patients were diagnosed as Mixed Dissociative Disorder and one case with conversion disorder. Dissociative disorders are characterized by a disruption and/or discontinuity in the normal integration of consciousness, memory, identity emotion, perception, body representation motor control, and behavior. Dissociative symptom can potentially disrupt every area of psychological functioning. Dissociative disorders have a high percentage of comorbidities like depression, PTSD, anxiety, self-mutilation, non-epileptic seizure and other psychiatric disorders. ${ }^{13}$ Bozkurt et al found high comorbid depression and PTSD in adolescents diagnosed with Dissociative disorder. ${ }^{14}$ In our case no.1, conversion symptoms witnessed might be an associated feature, but history revealed that in a previous occasion at least once the patient was diagnosed as conversion disorder. No associated physical illnesses were found in any of them. In case no. 4, concomitant migraine was present. All cases were given the diagnoses as per DSM 5 criteria. Dissociative Identity Disorder (300.14), Dissociative amnesia (300.12), Depersonalization/ Derealization (300.6), along with found.

All of our cases possess hysterical trait, all are adolescents, and there is a female preponderance, which is a common occurrence in Dissociative Disorders. It was noted that, most of the time, severity and intensity of the condition either aggravated or precipitated by detachment (actual or perceived) from the attachment figures. In all of our reported cases, we witnessed the events during their hospital stay.

Limitations: All of our reported cases did not present to us at the beginning of their symptoms. Physicians of different discipline were consulted for physical symptoms. Parents also sought for treatment from traditional and Spiritual healers. As it is not within the scope of this reporting, we did not provide the detailed psychiatric history and diagnostic issues which could be helpful for better understanding. We excluded malingering and factitious disorder through psychiatric assessment as it is known that those who feign dissociative symptoms remain undisturbed with their symptoms and they only report symptoms those are well known to other members of the society, yet definite absence of feigning could not be reliably determined, as no test or procedure is available to date. Could it be a self-injurious behaviour prior to hospital presentation, were not known.

Conclusion: Bloody tearing or haemolacria is an unusual complaint which concerns patients and guardians and many times may baffle physicians. In most of the cases reported so far, haemolacria accompanied bleeding from other body sites as well. This unusual condition has been described in patients with underlying pathology and more importantly sometimes without any known pathology. In the literature, haemolacria has been described as a 'rare' phenomenon, but we are in the opinion that this condition is not that uncommon, as similar cases are increasingly being reported than before. Many cases have been described as idiopathic from different countries. We suggest a multidisciplinary 
approach including psychiatric consultation for evaluation of the haemolacria patients when there is no known pathology. Although it is not recorded as an associated feature of Dissociative Disorders in any literature so far, we found bleeding through eyes in our cases of Dissociative disorders and conversion disorder, where no physical pathology were present,. However, it needs more case reports by psychiatrists. We reported physical symptom of bleeding through eyes in our cases of dissociative disorders and conversion disorder which cannot be explained by any standard criteria practiced at present.

\section{References:}

1. Ahluwalia, B.K., Khurana, A.K., and Sood, S., Bloody tears (haemolacria). Indian J. Ophthalmol. 1987; 35: 4143

2. Ho VH, Wilson MW, Linder JS, Fleming JC, Haik BG., "Bloody tears of unknown cause: case series and review of the literature." Ophthal Plast Reconstr Surg. 2004 Nov; 20(6):442-7.

3. Özcan KM, Özda ${ }^{\circ} \mathrm{T}$, Baran H, Ozdogan F, Dere H., "Haemolacria: Case report." International Journal of Pediatric Otorhinolaryngology. 2013 Jan; 77(1): 137138 .

4. Jason Z. S. Ho, Julian de Silva, and Jane Olver, "A Very Rare Case of Bloody Tears with Enigmatic Epistaxis and Haematuria." Orbit, 2011; 30(2), 116-117.

5. Verstraete M, "Psychogenic Haemorrhages.," Verh K Acad Geneeskd Belg. 1991; 53(1):5-28.
6. Gisele S. Silva, Patricia Nemoto, Paulo H. Monzillo, "Headache Bloody Tears, Gardner-Diamond Syndrome, and Trigemino-Autonomic Headache" Headache, 2014 Jan; 153-54 Available at www.researchgate. net/ publication/257750231_Bloody_Tears. (Accessed on 01/ 01/2015).

7. Wiese M F., "Bloody tears, and more! An unusual case of epistaxis." Br J Ophthalmol 2003;87:1051

8. Oyenusi EE, Ananti CH," Haemolacria (bloody tears): A perplexing symptom: A report of two cases" Niger J Paed 2015; 42 (1): $68-70$

9. Fessler D, (2002). "Starvation, serotonin, and symbolism. A psychobiocultural perspective on stigmata" Mind and Society: Cognitive Studies in Economics and Social Sciences 3 (2): 81-96. Retrieved on 30/05/2015

10. Wikipedia 2015. Available at http://en.wikipedia.org/wiki/ stigmata, accessed on 30/05/2015.

11. Wikipedia 2015. Available at http://en.wikipedia.org/wiki/ haemolacria, accessed on 18/04/2015

12. Renu Saxena et al. de Gruchy's Clinical Haematology in Medical Practice, $6^{\text {th }}$ adapted ed., India: The Magic International Pvt. Ltd. 2013.

13. American Psychiatric Association: Diagnostic and Statistical Manual of Mental Disorders, Fifth edition. Arlington, VA, American Psychiatric Association, 2013.

14. Bozucart, H.,Duzman Mutluer, T., Kose, C. and Zoroglu, S. (2015), High psychiatric comorbidity in adolescents with dissociative disorders. Psychiatry and Clinical Neuroscieces,69:369-374 doi:10.1111/pcn.12256 